\title{
Fatores intervenientes entre suporte social e autoeficácia em pessoas infectadas pelo HIV
}

\author{
Factors involved between social support and self-efficacy in HIV-infected individuals
}

\author{
Wendel Mombaque dos Santos ${ }^{1,3} \bowtie$, Stela Maris de Mello Padoin², Tânia Solange Bosi de Souza Magnago², \\ Graziele de Lima Dalmolin², Samuel Spierlberg Zuge ${ }^{3}$ \\ ${ }^{1}$ Hospital Universitário de Santa Maria. Santa Maria, RS \\ 2 Departamento de Enfermagem da Universidade Federal de Santa Maria (UFSM). Santa Maria, RS. \\ ${ }^{3}$ Programa de Pós-Graduação em Enfermagem da UFSM. Santa Maria, RS.
}

\section{RESUMO}

Objetivos: Verificar a relação entre a percepção do suporte social recebido e a expectativa de autoeficácia em adultos em tratamento antirretroviral para o HIV.

Métodos: Estudo transversal, realizado de janeiro a julho de 2012 no Ambulatório de Doenças Infecciosas do Hospital Universitário de Santa Maria, Rio Grande do Sul, com adultos em tratamento antirretroviral para HIV. Como instrumentos para coleta de dados foram aplicados um questionário com informações sociodemográficas e econômicas, a escala de expectativa de autoeficácia ao tratamento antirretroviral para o HIV e a escala para avaliação do suporte social. Utilizou-se estatística descritiva, correlação de Spearman e regressão logística multivariada. Foi considerado o nível de significância com valores iguais ou menores que $5 \%$.

Resultados: Verificou-se correlação entre suporte social emocional e expectativa de autoeficácia. O suporte social total apresenta como fator de risco contagem de linfócitos T CD4+ em até 50 células/ $\mu \mathrm{L}$ (odds ratio [OR] 1,17; intervalo de confiança [IC] 95\% 1,01-1,37) e como fator de proteção renda inferior a 16.024 reais anuais (OR 0,95; IC 95\% 0,91-0,99). A autoeficácia apresentou como fatores de proteção escolaridade entre quatro e sete anos de estudo (OR 0,94; IC 0,89-0,99), renda inferior a 16.024 reais anuais (OR 0,$91 ;$ IC $0,84-0,98)$, contagem de linfócitos T CD4+ em até 50 células/ $\mu \mathrm{L}$ (OR 0,84; IC 0,77-0,92), carga viral igual ou acima de 501 cópias/mL (OR 0,91; IC 0,87-0,95) e considerar o acompanhamento no serviço de saúde moderado (OR 0,96; IC 0,92-0,99) ou difícil (OR 0,94; IC 0,89-0,99).

Conclusões: O suporte social percebido associou-se com a expectativa de autoeficácia. Os dados sugerem que o suporte social e a expectativa de autoeficácia, além de afetar a resposta terapêutica ao tratamento, podem interferir no acompanhamento do serviço de saúde, assim como servir como barreira de proteção para manutenção da adesão ao TARV.

DESCRITORES: infecções por HIV; antirretrovirais; apoio social; autoeficácia.

\section{ABSTRACT}

Aims: To investigate the relationships between perceived social support received and expectation of self-efficacy.

Methods: Cross-sectional study conducted at the Outpatient Clinic of Infectious Diseases of the Teaching Hospital of Santa Maria, southern Brazil, from January to July 2012 with adults on antiretroviral treatment for HIV. To collect the data, we applied an instrument which included: a sociodemographic and economic questionnaire, a self-efficacy expectation scale for antiretroviral treatment, and the scale for evaluation of social support. We used descriptive statistics, Spearman's correlation, and multivariate logistic regression. Values equal to or less than 5\% were considered to be of statistical significance.

Results: There was a correlation between emotional social support and expectation of self-efficacy. Total social support presented CD4+ T lymphocyte count up to 50 cells $/ \mu \mathrm{L}$ (odds ratio $[\mathrm{OR}]=1.17 ; 95 \%$ confidence interval $[\mathrm{CI}]=1.01-1.37$ ) as a risk factor, and an income of less than 8,000 dollars per year $(\mathrm{OR}=0.95 ; 95 \% \mathrm{CI}=0.91-0.99)$ as a protective factor. Four to seven years of schooling $(\mathrm{OR}=0.94$; $95 \% \mathrm{CI}=0.89-0.99)$, income of less than 8,000 dollars per year $(\mathrm{OR}=0.91 ; 95 \% \mathrm{CI}=0.84-0.98)$, CD4+ T lymphocyte count up to 50 cells $/ \mu \mathrm{L}$ $(\mathrm{OR}=0.84 ; 95 \% \mathrm{CI}=0.77-0.92)$, viral load equal to or greater than $501 \mathrm{copies} / \mathrm{mL}(\mathrm{OR}=0.91 ; 95 \% \mathrm{CI}=0.87-0.95)$, and healthcare follow-up considered moderate $(\mathrm{OR}=0.96 ; 95 \% \mathrm{CI}=0.92-0.99)$ or difficult $(\mathrm{OR}=0.94 ; 95 \% \mathrm{CI}=0.89-0.99)$ were regarded as protective factors for self-efficacy.

Conclusions: Perceived social support is associated with expectation of self-efficacy. The data suggest that social support and expectation of self-efficacy, in addition to affecting the therapeutic response to treatment, may interfere with healthcare follow-up and act as a protective factor for sustained adherence to antiretroviral therapy.

KEY WORDS: HIV infections; anti-retroviral agents; social support; self efficacy. 
Abreviaturas: HIV: Vírus da Imunodeficiência Humana ; TARV: tratamento antirretroviral; HUSM: Hospital Universitário de Santa Maria ; OR: odds ratio

\section{INTRODUÇÃO}

Aproximadamente 30 milhões de pessoas estão infectadas pelo vírus da imunodeficiência adquirida (HIV) no mundo, dentre as quais 28,6 milhões são elegíveis para realizar o tratamento antirretroviral (TARV) e 1,6 milhões morrem anualmente em função da ausência ou não adesão a esse tratamento. No Brasil, $74 \%$ das pessoas infectadas estão vinculados a serviços de saúde e monitorando o tratamento [1-2].

$\mathrm{O}$ TARV proporciona sobrevida às pessoas infectadas pelo HIV por meio da supressão da carga viral e aumento de linfócitos T CD4+ na corrente sanguínea. A eficácia do tratamento está relacionada à sua adesão, no entanto, sua manutenção durante todo o tratamento é um problema [3-5]. A qualidade dos serviços de saúde, os custos relacionados com o TARV e a manutenção das redes de apoio social podem ser afetadas pelo estigma relacionado ao HIV, constituindo-se fatores que influenciam a adesão e tornando necessária a manutenção do equilíbrio entre a saúde física com a integridade social [6].

A adesão ao TARV está relacionada com carga viral, contagem de linfócitos T CD4+, complexidade do tratamento e quantidade de pílulas ingeridas diariamente. Fatores relacionados ao estresse, depressão e ansiedade atuam como fatores de risco para perda da adesão, enquanto o apoio social consiste em fator de proteção [7]. Como dados que podem mensurar a adesão ao TARV podem ser citados a expectativa de autoeficácia e o suporte social percebido [7-10].

A expectativa de autoeficácia representa o julgamento da pessoa infectada sobre sua própria capacidade de cumprir com sucesso o tratamento medicamentoso através do seguimento regular da prescrição, ou seja, sua capacidade de ingerir os medicamentos nos horários indicados e quantidades corretas [11-13]. O suporte social consiste na satisfação da pessoa com o apoio que tem recebido da rede de relações sociais, ou seja, de que forma as pessoas oferecerem condições que auxiliam no TARV, e pode ser dividido em duas categorias: o suporte social instrumental e o suporte social emocional. O primeiro, refere-se à disponibilização de ajuda em situações operacionais do cotidiano, tais como apoio financeiro e/ou material; o segundo, aos comportamentos de escutar, de prover atenção ou fazer companhia para que a pessoa infectada pelo HIV possa se sentir estimada e/ou cuidada [14-16].

Não há evidências concretas de como o suporte social se relaciona com a expectativa de autoeficácia e quais os fatores que podem interferir como risco ou proteção para os mesmos; apenas há evidencias da influência destes com a adesão ao TARV [17-19]. Portanto, o presente estudo tem como objetivo verificar a relação entre o suporte social e a autoeficácia em pacientes adultos em TARV para o HIV no Hospital Universitário de Santa Maria, Rio Grande do Sul, Brasil.

\section{MÉTODOS}

Trata-se de um estudo com delineamento transversal, realizado no Ambulatório de Doenças Infecciosas do Hospital Universitário de Santa Maria (HUSM), no período de janeiro a julho de 2012, com adultos em TARV para o HIV. A pesquisa respeitou todos os preceitos éticos, e previamente à coleta de dados todos os participantes assinaram o termo de consentimento livre e esclarecido, o qual foi aprovado pelo Comitê de Ética em Pesquisa da Universidade Federal de Santa Maria, pelo processo número 23081.015120/2011-15.

Os critérios de inclusão foram idade igual ou superior a 20 anos e cadastro pelo menos há três meses na unidade de distribuição de medicação do HUSM. Os critérios de exclusão foram limitação cognitiva ou mental; estar em regime penitenciário; e mulheres no período gravídico-puerperal. O cálculo amostral foi realizado com precisão de 5,7\% e intervalo de confiança de $95 \%$, a partir de uma população de 432 pessoas, resultando em uma amostra mínima de 179 participantes, sendo selecionados conforme a chegada ao serviço.

Foram consideradas variáveis dependentes a expectativa de autoeficácia ao TARV [13] e a escala para avaliação do suporte social em HIV/AIDS [14]. A escala de avaliação da expectativa de autoeficácia compreendeu uma escala unifatorial composta por 21 itens de respostas tipo Likert de cinco pontos $(0=\mathrm{com}$ certeza não vou tomar; 1 = acho que não vou tomar; 2 = não sei; 3 = acho que vou tomar; $4=$ com certeza vou tomar). Esses escores propõem uma variável contínua e podem variar de zero a 100 , de modo que os valores elevados indicam melhor expectativa de autoeficácia.

A escala de avaliação do suporte social foi bidimensional composta de 24 itens. A primeira dimensão 
estava relacionada ao suporte emocional (12 itens) e a segunda ao suporte instrumental (12 itens). As respostas foram também em Escala tipo Likert de cinco pontos tanto para disponibilidade de suporte $(1=$ nunca; $2=$ raramente; $3=$ às vezes $; 4=$ frequentemente $;=$ sempre) quanto para satisfação com o suporte $(1=$ muito insatisfeito; 2 = insatisfeito; $3=$ nem satisfeito/nem insatisfeito; $4=$ satisfeito; $5=$ muito satisfeito), tendo amplitude de escore geral de 24 a 130 pontos; quanto maior a pontuação melhor o suporte social.

As variáveis independentes foram sexo, raça, idade, grau de instrução, renda anual, tempo de diagnóstico, tempo de tratamento, quantidade de comprimidos antirretrovirais tomados por dia, contagem de células T CD4+, valor da carga viral, conhecimento da sua infecção pelo HIV pelos colegas de trabalho e avaliação sobre a facilidade ou dificuldade de manutenção do acompanhamento no serviço de saúde.

Utilizou-se estatística descritiva para apresentação das características basais da população e teste do qui-quadrado de Pearson ou exato de Fischer nas associações bivariadas. $\mathrm{O}$ teste de correlação de Spearman foi empregado para avaliar relação entre expectativa de autoeficácia e suporte social, conforme as características basais da população, sendo classificada em fraca $(r=0,00-0,30)$, moderada $(\mathrm{r}=0,31-0,70)$ ou forte $(\mathrm{r}=0,71-1,00)$. A regressão logística multinomial foi usada para obter estimativas de OR e seus respectivos intervalos de confiança (IC 95\%) para o conjunto de fatores intervenientes. Para a comparação de médias entre expectativa de autoeficácia e suporte social conforme as características basais da população, foram utilizados os testes Kruskal-Wallis ou U de Mann-Whitney. Em todas as análises, adotou-se nível de significância $p<0,05$. A organização dos dados foi realizada no programa Epi Info 6.04, com dupla digitação independente. Após verificação e correção das inconsistências, a análise dos dados foi feita utilizando-se o software IBM SPSS versão 21 .

\section{RESULTADOS}

Participaram do estudo 179 adultos em TARV para o HIV, dos quais 91 eram homens e 88 mulheres. As características basais dos participantes do estudo podem ser observadas na Tabela 1.

Ao serem realizadas as análises conforme os estratos da população, foram verificadas as seguintes correlações significativas entre suporte social emocional e autoeficácia: correlação moderada positiva com idade entre 35 a 39 anos; correlação fraca positiva com escolaridade entre um a três anos de estudo; correlação fraca positiva com renda até 16.024 reais ano; correlação moderada positiva com tempo de diagnóstico menor ou igual a cinco anos; correlação fraca positiva com tempo de tratamento menor ou igual a 5 anos; correlação forte positiva com contagem de células T CD4 menor ou igual a 50 células $/ \mu \mathrm{L}$; correlação moderada positiva com contagem de células T CD4 entre 51 a 200 células/ $\mu \mathrm{L}$; correlação fraca positiva com carga viral acima ou igual a 501 cópias/ $\mathrm{mL}$; e correlação moderada positiva com conhecimento sobre a infecção no trabalho (Tabela 2).

Mantendo os mesmos estratos, foi verificada correlação significativa entre suporte social instrumental e autoeficácia somente na raça negra, com correlação moderada positiva $(\mathrm{p}=0,05 ; \mathrm{r}=0,39)$, ou seja, quanto maior o suporte social instrumental recebido maior foi a expectativa de autoeficácia (Tabela 2).

$\mathrm{Na}$ análise entre suporte social total e expectativa de autoeficácia, as seguintes estratificações apresentaram resultados significativos: correlação moderada positiva com raça negra, correlação fraca com escolaridade entre um e três anos de estudo e correlação moderada positiva com escolaridade entre quatro e sete anos de estudo (Tabela 2).

Ao ser realizada a análise independente da estratificação da amostra, somente o suporte social emocional apresentou resultado significativo com a expectativa de autoeficácia, com uma correlação fraca positiva, ou seja, quanto maior o suporte social emocional recebido maior foi a expectativa de autoeficácia em todos os estratos da amostra (Tabela 2).

A análise da distribuição dos níveis de suporte social e autoeficácia, conforme as características basais da amostra, demonstra que houve diferença significativa no suporte instrumental conforme a renda dos participantes: aqueles com renda anual até 16.024 reais anuais possuíam média de 16,66 pontos superior quando comparados aos que possuíam renda anual superior a 16.024 reais (Tabela 3).

Foi verificada significância estatística nas seguintes características da amostra quando associadas à distribuição da expectativa de autoeficácia. Idade: os grupos limites (idade entre 20 e 29 anos e acima de 50 anos) apresentaram maior chance de possuir expectativa de autoeficácia. Renda: as pessoas com renda superior a 16.024 reais/ano possuíam cerca de 25 vezes maior chance de expectativa de autoeficácia do que aquelas com renda inferior. Contagem de células T CD4+: a probabilidade de possuir expectativa de autoeficácia era três vezes superior no grupo com valores superiores a 350 células/ $\mu \mathrm{L}$, quando comparado 
ao com valores inferiores a 50 células/ $\mu \mathrm{L}$ e 15 vezes superior quando comparado ao grupo com valores de células T CD4+ entre 51 e 350 células $/ \mu \mathrm{L}$. Carga viral: as pessoas com valores inferiores a 50 cópias/ $\mathrm{mL}$ apresentaram cerca de 42 vezes mais chance de expectativa de autoeficácia quando comparadas às que possuíam mais de 501 cópias $/ \mathrm{mL}$ e 26 vezes a mais em relação às que possuíam entre $51-500$ cópias $/ \mathrm{mL}$ (Tabela 4).

Ao ser realizada a regressão logística multinomial foram verificados fatores que elevam o suporte social e a expectativa de autoeficácia (OR com valores superiores a um), assim como fatores de redução dos mesmos (OR com valores inferiores a um) (Tabela 4).

O acompanhamento nos serviços de saúde, quando considerado moderado, foi fator de redução do suporte social emocional. A chance de haver suporte instrumental era 1,12 vezes maior quando a renda era de até 16.024 reais anuais. A redução do suporte social total associou-se à menor renda: quando esta era de até 16.024 reais anuais, a chance de haver suporte social era 0,95 vezes menor. Já a contagem de células T CD4+ em até 50 células $/ \mu \mathrm{L}$ eleva a chance de suporte social total em 1,17 vezes (Tabela 4).

A expectativa de autoeficácia somente apresentou associação significativa com fatores de risco para sua redução; entre estes estão escolaridade entre quatro e sete anos de estudo, renda de até 16.024 reais anuais, contagem de células T CD4+ em até 50 células $/ \mu \mathrm{L}$, carga viral igual ou acima a 501 cópias $/ \mathrm{mL}$ e considerar o acompanhamento no serviço de saúde difícil (Tabela 4).

$\mathrm{O}$ teste $\mathrm{U}$ de Mann-Whitney indicou diferença significativa entre o grupo com suporte social fornecido por familiares que residem com o paciente, tanto no suporte emocional $(25$ pontos a mais, $p=0,002)$ quanto instrumental ( 30 pontos a mais, $p<0,001$ ), e o grupo que não recebia essa fonte de suporte social. As demais fontes de suporte social, apesar de não demonstrarem resultados significativos, apresentaram valores mais elevados de suporte social percebido quando havia alguma fonte de suporte, excetuando-se quando este era fornecido por amigos.

Tabela 1. Características basais de adultos infectados pelo HIV, atendidos no Hospital Universitário de Santa Maria, no ano de 2012. Santa Maria, RS, Brasil.

\begin{tabular}{|c|c|}
\hline Características & $\begin{array}{l}\text { Participantes } \\
\quad(n=179)\end{array}$ \\
\hline Sexo masculino (\%) & 50,8 \\
\hline Raça branca (\%) & 73,2 \\
\hline Idade em anos, mediana (min-max) & $42(22-77)$ \\
\hline Instrução em anos, mediana (min-max) & $6(0-15)$ \\
\hline Renda em real anual, mediana (min-max) & $16.024(2.436-244.494)$ \\
\hline Tempo de diagnóstico em meses, mediana (min-max) & $84(3-288)$ \\
\hline Tempo de tratamento em meses, mediana (min-max) & $72(3-240)$ \\
\hline Comprimidos ART tomados por dia, mediana (min-max) & $4(1-19)$ \\
\hline Valor células T CD4+ em células/ $\mu \mathrm{L}$, mediana (min-max) & $469(6-1.269)$ \\
\hline Valor Carga Viral em cópias/ml, mediana (min-max) & $0(0-345.546)$ \\
\hline Conhecimento da infecção por colegas de trabalho (\%) & 46 \\
\hline Considerava fácil manter o acompanhamento no serviço de saúde (\%) & 58,1 \\
\hline Avaliação do suporte social emocional em pontos, mediana (min-max) & $47,79(12-60)$ \\
\hline Avaliação do suporte social instrumental em pontos, mediana (min-max) & $42,47(13-60)$ \\
\hline Avaliação do suporte social total em pontos, mediana (min-max) & $89,86(25-120)$ \\
\hline Expectativa de autoeficácia em pontos, mediana (min-max) & $99,03(50-100)$ \\
\hline
\end{tabular}


Tabela 2. Correlação entre suporte social e suas dimensões com a expectativa de autoeficácia conforme características basais da amostra.

\begin{tabular}{|c|c|c|c|c|c|c|}
\hline \multirow{3}{*}{ Variáveis } & \multicolumn{6}{|c|}{ Correlação } \\
\hline & \multicolumn{2}{|c|}{$\begin{array}{l}\text { Suporte emocional } \\
\text { x autoeficácia }\end{array}$} & \multicolumn{2}{|c|}{$\begin{array}{l}\text { Suporte instrumental } \\
\mathrm{x} \text { autoeficácia }\end{array}$} & \multicolumn{2}{|c|}{$\begin{array}{c}\text { Suporte social total } \\
\text { x autoeficácia }\end{array}$} \\
\hline & $\mathbf{r}$ & $\mathbf{P}$ & $\mathbf{r}$ & $\mathbf{P}$ & $\mathbf{r}$ & $\mathbf{P}$ \\
\hline \multicolumn{7}{|l|}{ Sexo } \\
\hline Masculino & 0,15 & 0,13 & 0,05 & 0,58 & 0,12 & 0,23 \\
\hline Feminino & 0,13 & 0,20 & $-0,01$ & 0,86 & 0,09 & 0,40 \\
\hline \multicolumn{7}{|l|}{ Raça } \\
\hline Branca & 0,09 & 0,25 & $-0,04$ & 0,64 & 0,04 & 0,65 \\
\hline Negra & 0,33 & 0,11 & 0,39 & 0,05 & 0,48 & 0,01 \\
\hline Outra & 0,12 & 0,56 & $-0,16$ & 0,44 & 0,01 & 0,95 \\
\hline \multicolumn{7}{|l|}{ Idade } \\
\hline 20-29 anos & $-0,14$ & 0,96 & $-0,01$ & 0,96 & 0,60 & 0,82 \\
\hline 30-39 anos & 0,35 & 0,007 & 0,10 & 0,44 & 0,22 & 0,08 \\
\hline 40-49 anos & $-0,00$ & 0,97 & $-0,63$ & 0,62 & $-0,02$ & 0,81 \\
\hline$\geq 50$ anos & 0,11 & 0,48 & 0,05 & 0,71 & 0,09 & 0,54 \\
\hline \multicolumn{7}{|l|}{ Escolaridade } \\
\hline Nenhum ano de estudo & 0,25 & 0,57 & 0,37 & 0,93 & 0,11 & 0,81 \\
\hline 1-3 anos de estudo & 0,28 & 0,01 & 0,18 & 0,11 & 0,25 & 0,02 \\
\hline 4 e 7 anos de estudo & 0,66 & 0,04 & 0,28 & 0,27 & 0,55 & 0,02 \\
\hline$\geq 8$ anos de estudo & $-0,14$ & 0,20 & $-0,19$ & 0,08 & $-0,15$ & 0,17 \\
\hline \multicolumn{7}{|l|}{ Renda } \\
\hline$<16.024$ reais anuais & 0,18 & 0,04 & 0,09 & 0,27 & 0,16 & 0,06 \\
\hline$\geq 16.024$ reais anuais & $-0,13$ & 0,36 & $-0,21$ & 0,14 & $-0,15$ & 0,28 \\
\hline \multicolumn{7}{|l|}{ Tempo de diagnóstico } \\
\hline$\leq 5$ anos & 0,32 & $<0,01$ & $-0,03$ & 0,76 & 0,16 & 0,21 \\
\hline 6-10 anos & $-0,1$ & 0,4 & $-0,12$ & 0,31 & $-0,12$ & 0,32 \\
\hline 11-15 anos & 0,28 & 0,08 & 0,19 & 0,23 & 0,25 & 0,10 \\
\hline$\geq 16$ anos & $-0,33$ & 0,31 & $-0,21$ & 0,52 & $-0,28$ & 0,38 \\
\hline \multicolumn{7}{|l|}{ Tempo de tratamento } \\
\hline$\leq 5$ anos & 0,23 & 0,03 & 0,03 & 0,77 & 0,14 & 0,17 \\
\hline 6-10 anos & $-0,07$ & 0,56 & $-0,12$ & 0,30 & $-0,1$ & 0,39 \\
\hline $11-15$ anos & $-0,11$ & 0,61 & $-0,15$ & 0,47 & $-0,14$ & 0,51 \\
\hline$\geq 16$ anos & $-0,55$ & 0,44 & $-0,35$ & 0,64 & $-0,49$ & 0,50 \\
\hline \multicolumn{7}{|l|}{ Comprimidos } \\
\hline$\leq 4$ comprimidos/dia & 0,14 & 0,14 & $-0,04$ & 0,65 & 0,06 & 0,50 \\
\hline$\geq 5$ comprimidos/dia & 0,14 & 0,21 & 0,13 & 0,24 & 0,15 & 0,17 \\
\hline \multicolumn{7}{|l|}{ Contagem de Células T CD4+ ${ }^{+}$} \\
\hline$\leq 50$ células $/ \mu \mathrm{L}$ & 0,96 & 0,00 & 0,50 & 0,24 & 0,71 & 0,07 \\
\hline $51-200$ células $/ \mu \mathrm{L}$ & 0,41 & 0,02 & 0,14 & 0,47 & 0,31 & 0,10 \\
\hline 201-350 células $/ \mu \mathrm{L}$ & 0,59 & 0,78 & $-0,19$ & 0,36 & $-0,07$ & 0,73 \\
\hline$\geq 350$ células $/ \mu \mathrm{L}$ & 0,08 & 0,38 & 0,01 & 0,85 & 0,06 & 0,50 \\
\hline \multicolumn{7}{|l|}{ Carga Viral } \\
\hline ～ 50 cópias $/ \mathrm{mL}$ & 0,07 & 0,41 & 0,02 & 0,77 & 0,05 & 0,50 \\
\hline 51-500 cópias/mL & 0,39 & 0,20 & 0,12 & 0,70 & 0,29 & 0,35 \\
\hline$\geq 501$ cópias $/ \mathrm{mL}$ & 0,37 & 0,02 & 0,03 & 0,86 & 0,22 & 0,19 \\
\hline \multicolumn{7}{|c|}{ Conhecimento sobre a doença no trabalho } \\
\hline Sim & 0,44 & $<0,01$ & 0,19 & 0,32 & 0,37 & 0,05 \\
\hline Não & 0,00 & 0,97 & $-0,12$ & 0,49 & $-0,06$ & 0,71 \\
\hline \multicolumn{7}{|c|}{ Acompanhamento no serviço de saúde } \\
\hline Fácil & 0,16 & 0,09 & 0,10 & 0,30 & 0,14 & 0,13 \\
\hline Moderado & 0,08 & 0,53 & $-0,12$ & 0,35 & $-0,00$ & 0,96 \\
\hline Difícil & 0,16 & 0,58 & 0,08 & 0,78 & 0,13 & 0,65 \\
\hline Independente de estratificação & 0,14 & 0,05 & 0,02 & 0,76 & 0,10 & 0,16 \\
\hline
\end{tabular}

$r=$ Correlação de Spearman. 
Tabela 3. Distribuição das características basais da população conforme suporte social.

\begin{tabular}{|c|c|c|c|c|c|c|c|c|}
\hline \multirow{3}{*}{ Características da amostra } & \multicolumn{6}{|c|}{ Suporte Social } & \multirow{2}{*}{\multicolumn{2}{|c|}{ Autoeficácia }} \\
\hline & \multicolumn{2}{|c|}{ Emocional } & \multicolumn{2}{|c|}{ Instrumental } & \multicolumn{2}{|c|}{ Total } & & \\
\hline & Mean rank & p & Mean rank & p & Mean rank & $\mathbf{p}$ & Mean rank & $\mathbf{p}$ \\
\hline Sexo** & & 0,27 & & 0,77 & & 0,48 & & 0,71 \\
\hline Masculino & 94,14 & & 91,07 & & 92,63 & & 88,71 & \\
\hline Feminino & 85,72 & & 88,89 & & 87,28 & & 91,34 & \\
\hline Raça* & & 0,33 & & 0,16 & & 0,18 & & 0,46 \\
\hline Branca & 89,97 & & 91,63 & & 90,56 & & 92,50 & \\
\hline Negra & 101,21 & & 98,71 & & 102,10 & & 86,35 & \\
\hline Outra & 78,96 & & 72,42 & & 74,81 & & 79,98 & \\
\hline Idade* & & 0,95 & & 0,65 & & 0,87 & & 0,02 \\
\hline 20-29 anos & 88,69 & & 100,13 & & 94,59 & & 102,41 & \\
\hline 30-39 anos & 91,91 & & 91,94 & & 91,68 & & 74,98 & \\
\hline $40-49$ anos & 91,02 & & 90,72 & & 90,92 & & 92,07 & \\
\hline$\geq 50$ anos & 86,23 & & 82,22 & & 84,41 & & 102,76 & \\
\hline Escolaridade* & & 0,51 & & 0,34 & & 49 & & 0,18 \\
\hline Nenhum ano de estudo & 80,50 & & 111,93 & & 96,50 & & 72,64 & \\
\hline 1-3 anos de estudo & 96,60 & & 94,61 & & 96,25 & & 87,27 & \\
\hline 4 e 7 anos de estudo & 87,56 & & 92,00 & & 87,97 & & 74,62 & \\
\hline$\geq 8$ anos de estudo & 84,87 & & 83,04 & & 83,69 & & 97,61 & \\
\hline Renda** & & 0,54 & & 0,05 & & 0,43 & & 0,002 \\
\hline$<16.024$ reais anuais & 86,10 & & 92,01 & & 89,32 & & 80,74 & \\
\hline$\geq 16.024$ reais anuais & 91,29 & & 75,35 & & 82,59 & & 105,77 & \\
\hline Tempo de diagnóstico* & & 0,39 & & 0,53 & & 0,48 & & 0,63 \\
\hline$\leq 5$ anos & 96,93 & & 98,04 & & 97,74 & & 95,21 & \\
\hline 6-10 anos & 90,78 & & 85,93 & & 88,48 & & 89,57 & \\
\hline $11-15$ anos & 78,79 & & 86,59 & & 81,71 & & 86,46 & \\
\hline$\geq 16$ anos & 88,14 & & 83,73 & & 87,32 & & 77,14 & \\
\hline Tempo de tratamento* & & 0,70 & & 0,67 & & 0,72 & & 0,49 \\
\hline$\leq 5$ anos & 91,71 & & 93,76 & & 93,09 & & 93,51 & \\
\hline $6-10$ anos & 89,82 & & 84,25 & & 87,03 & & 86,69 & \\
\hline $11-15$ anos & 78,72 & & 86,67 & & 81,17 & & 77,46 & \\
\hline$\geq 16$ anos & 77,25 & & 78,25 & & 79,00 & & 97,13 & \\
\hline Comprimidos** & & 0,74 & & 0,28 & & 0,62 & & 0,14 \\
\hline$\leq 4$ comprimidos/dia & 91,09 & & 86,40 & & 88,33 & & 94,50 & \\
\hline$\geq 5$ comprimidos/dia & 88,55 & & 94,77 & & 92,21 & & 84,05 & \\
\hline Contagem de Células T CD4 ${ }^{+*}$ & & 0,30 & & 0,85 & & 0,52 & & 0,004 \\
\hline$\leq 50$ células $/ \mu \mathrm{L}$ & 106,64 & & 101,64 & & 104,79 & & 32,21 & \\
\hline 51-200 células/ $\mu \mathrm{L}$ & 74,02 & & 83,43 & & 78,52 & & 81,93 & \\
\hline 201-350 células $/ \mu \mathrm{L}$ & 94,06 & & 89,77 & & 91,79 & & 81,00 & \\
\hline$\geq 350$ células $/ \mu \mathrm{L}$ & 91,21 & & 90,16 & & 90,72 & & 96,25 & \\
\hline Carga Viral* & & 0,80 & & 0,93 & & 0,98 & & 0,000 \\
\hline > 50 cópias $/ \mathrm{mL}$ & 89,09 & & 89,04 & & 89,33 & & 99,59 & \\
\hline 51-500 cópias/mL & 98,88 & & 87,00 & & 91,88 & & 73,17 & \\
\hline Z 501 cópias/mL & 87,81 & & 92,09 & & 89,31 & & 57,34 & \\
\hline Conhecimento da infecção no trabalho ${ }^{1 * *}$ & & 0,63 & & 0,28 & & 0,49 & & 0,28 \\
\hline Sim & 32,71 & & 34,18 & & 33,21 & & 34,18 & \\
\hline Não & 30,50 & & 29,29 & & 30,09 & & 29,29 & \\
\hline Acompanhamento no serviço de saúde* & & 00,39 & & 00,06 & & 00,28 & & 0,09 \\
\hline Fácil & 80,32 & & 95,19 & & 97,09 & & 93,56 & \\
\hline Moderado & 79,18 & & 82,73 & & 79,73 & & 83,18 & \\
\hline Difícil & 97,65 & & 83,11 & & 82,07 & & 93,25 & \\
\hline
\end{tabular}

* Teste de Kruskal-wallis; ** Teste U de mann-whitney.

'número de sujeitos igual a 62. 
Tabela 4. Fatores de risco para percepção de suporte social e expectativa de autoeficácia, em adultos em tratamento antirretroviral para o HIV, janeiro a julho de 2012, Ambulatório de Doenças Infecciosas do Hospital Universitário de Santa Maria, Rio Grande do Sul, Brasil.

\begin{tabular}{|c|c|c|c|c|}
\hline \multirow[b]{2}{*}{ Características da amostra } & \multicolumn{3}{|c|}{ Percepção de suporte social } & \multirow{2}{*}{$\begin{array}{l}\text { Expectativa de } \\
\text { autoeficácia } \\
\text { OR (IC)* }\end{array}$} \\
\hline & $\begin{array}{l}\text { Emocional } \\
\text { OR (IC)** }\end{array}$ & $\begin{array}{l}\text { Instrumental } \\
\text { OR (IC)* }\end{array}$ & $\begin{array}{c}\text { Total } \\
\text { OR (IC)* }\end{array}$ & \\
\hline \multicolumn{5}{|l|}{ Sexo } \\
\hline Masculino (referência) & 1 & 1 & 1 & 1 \\
\hline Feminino & $0,99(0,96-1,01)$ & $0,99(0,96-1,02)$ & $0,98(0,94-1,02)$ & $1,00(0,97-1,04)$ \\
\hline \multicolumn{5}{|l|}{ Raça } \\
\hline Branca (referência) & 1 & 1 & 1 & 1 \\
\hline Negra & $1,02(0,97-1,06)$ & $0,96(0,86-1,07)$ & $1,02(0,96-1,08)$ & $1,00(0,94-1,06)$ \\
\hline Outra & $0,98(0,94-1,02)$ & $0,93(0,84-1,04)$ & $1,01(0,96-1,07)$ & $0,97(0,93-1,01)$ \\
\hline \multicolumn{5}{|l|}{ Idade } \\
\hline 20-29 anos (referência) & 1 & 1 & 1 & 1 \\
\hline 30-39 anos & $1,01(0,96-1,06)$ & $0,88(0,76-1,01)$ & $1,06(0,98-1,14)$ & $0,92(0,85-1,00)$ \\
\hline 40-49 anos & $1,00(0,95-1,06)$ & $0,91(0,79-1,04)$ & $1,04(0,97-1,12)$ & $0,98(0,90-1,07)$ \\
\hline$\geq 50$ anos & $0,99(0,94-1,05)$ & $0,88(0,76-1,02)$ & $1,04(0,97-1,13)$ & $1,00(0,91-1,10)$ \\
\hline \multicolumn{5}{|l|}{ Escolaridade } \\
\hline Nenhum ano de estudo & $1,00(0,93-1,07)$ & $1,17(0,96-1,43)$ & $0,94(0,85-1,04)$ & $0,96(0,88-1,03)$ \\
\hline 1-3 anos de estudo & $1,01(0,98-1,04)$ & $0,99(0,92-1,07)$ & $1,01(0,97-1,05)$ & $0,96(0,93-1,01)$ \\
\hline 4-7 anos de estudo & $1,01(0,96-1,06)$ & $1,01(0,88-1,15)$ & $1,00(0,93-1,08)$ & $0,94(0,89-0,99)$ \\
\hline$\geq 8$ anos de estudo (referência) & 1 & 1 & 1 & 1 \\
\hline \multicolumn{5}{|l|}{ Renda } \\
\hline$<16.024$ reais anuais & $0,99(0,96-1,02)$ & $1,12(1,02-1,22)$ & $0,95(0,91-0,99)$ & $0,91(0,84-0,98)$ \\
\hline$\geq 16.024$ reais anuais (referência) & 1 & 1 & 1 & 1 \\
\hline \multicolumn{5}{|l|}{ Tempo de diagnóstico } \\
\hline$\leq 5$ anos (referência) & 1 & 1 & 1 & 1 \\
\hline $6-10$ anos & $0,97(0,94-1,01)$ & $0,99(0,91-1,07)$ & $0,98(0,94-1,03)$ & $1,01(0,97-1,05)$ \\
\hline 11-15 anos & $0,97(0,93-1,01)$ & $1,02(0,92-1,13)$ & $0,97(0,92-1,02)$ & $0,99(0,95-1,04)$ \\
\hline$\geq 16$ anos & $0,98(0,92-1,04)$ & $0,98(0,84-1,14)$ & $0,99(0,91-1,08)$ & $1,02(0,94-1,12)$ \\
\hline \multicolumn{5}{|l|}{ Tempo de tratamento } \\
\hline$\leq 5$ anos (referência) & 1 & 1 & 1 & 1 \\
\hline $6-10$ anos & $0,99(0,96-1,02)$ & $0,96(0,89-1,04)$ & $1,00(0,96-1,05)$ & $1,01(0,97-1,05)$ \\
\hline 11-15 anos & $0,98(0,94-1,02)$ & $1,02(0,91-1,14)$ & $0,98(0,92-1,04)$ & $1,02(0,96-1,08)$ \\
\hline$\geq 16$ anos & $0,97(0,89-1,06)$ & $1,02(0,96-1,08)$ & $0,97(0,85-1,10)$ & $1,12(0,83-1,50)$ \\
\hline \multicolumn{5}{|l|}{ Comprimidos } \\
\hline$\leq 4$ comprimidos/dia (referência) & 1 & 1 & 1 & 1 \\
\hline$\geq 5$ comprimidos/dia & $0,99(0,97-1,02)$ & $1,04(0,97-1,12)$ & $0,98(0,94-1,02)$ & $0,96(0,93-1,00)$ \\
\hline \multicolumn{5}{|l|}{ Contagem de Células T CD4 } \\
\hline$\leq 50$ células $/ \mu \mathrm{L}$ & $1,03(0,95-1,12)$ & $0,84(0,66-1,08)$ & $1,17(1,01-1,37)$ & $0,84(0,77-0,92)$ \\
\hline 51-200 células/ $\mu \mathrm{L}$ & $0,97(0,93-1,00)$ & $1,05(0,94-1,16)$ & $0,96(0,91-1,01)$ & $0,98(0,93-1,03)$ \\
\hline 201-350 células $/ \mu \mathrm{L}$ & $1,00(0,96-1,04)$ & $0,96(0,86-1,07)$ & $1,01(0,96-1,07)$ & $0,97(0,92-1,03)$ \\
\hline$\geq 351$ células/ $\mu \mathrm{L}$ (referência) & 1 & 1 & 1 & 1 \\
\hline \multicolumn{5}{|l|}{ Carga Viral } \\
\hline ～ 50 cópias/ mL (referência) & 1 & 1 & 1 & 1 \\
\hline 51-500 cópias/ mL & $1,01(0,95-1,07)$ & $0,94(0,81-1,08)$ & $1,03(0,95-1,11)$ & $0,93(0,88-1,00)$ \\
\hline 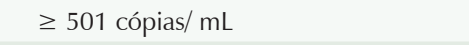 & $0,99(0,96-1,03)$ & $0,99(0,90-1,09)$ & $1,00(0,95-1,06)$ & $0,91(0,87-0,95)$ \\
\hline \multicolumn{5}{|l|}{ Conhecimento sobre a doença no trabalho } \\
\hline Sim (referência) & 1 & 1 & 1 & 1 \\
\hline Não & $0,97(0,93-1,02)$ & $0,96(0,84-1,09)$ & $1,00(0,93-1,07)$ & $0,97(0,90-1,04)$ \\
\hline \multicolumn{5}{|l|}{ Acompanhamento no serviço de saúde } \\
\hline Fácil (referência) & 1 & 1 & 1 & 1 \\
\hline Moderado & $0,96(0,93-1,00)$ & $1,02(0,94-1,10)$ & $0,97(0,93-1,01)$ & $0,96(0,92-0,99)$ \\
\hline Difícil & $0,96(0,92-0,99)$ & $0,99(0,86-1,13)$ & $0,98(0,91-1,06)$ & $0,94(0,89-0,99)$ \\
\hline
\end{tabular}

* Teste do qui quadrado ou Exato de Fisher. 


\section{DISCUSSÃO}

Neste estudo foi verificada a correlação entre o suporte social emocional e expectativa de autoeficácia, concordando com as evidências que sugerem que a autoeficácia pode ser afetada pelo suporte social total e suas subcategorias [17-19]. A correlação verificada, somente com o suporte social emocional, justifica-se por estar presente em 10 estratos da população, ao passo que o suporte social instrumental e total apresentaram, apenas, uma e três correlações respectivamente.

A correlação apresentada entre suporte social instrumental e suporte social total com autoeficácia na raça negra pode ser explicada, principalmente, por possuírem a maior concentração da sua população com menor renda, quando comparada às outras raças. Essas pessoas foram também as que apresentaram maior percepção do suporte social fornecido. Dados de outros estudos realizados no Brasil apontam que a raça negra possui renda inferior quando comparada às demais, além de possuir risco de não adesão [20,21], evidenciando a necessidade do suporte instrumental para a manutenção do seu TARV.

A autoeficácia foi correlacionada ao suporte social emocional na população entre 35 e 39 anos, corroborando com dados de outro estudo que demonstrou que a idade interfere negativamente no apoio social percebido de pessoas infectadas pelo HIV [10], de forma que quanto maior a idade menor será a expectativa de autoeficácia do tratamento. Aliado a este fato, essa faixa de população foi a que apresentou a menor média de expectativa de autoeficácia, necessitando mais do apoio emocional fornecido para manter o tratamento, sendo evidenciado pela associação entre a fonte de apoio proveniente de familiares que residem com o paciente.

Os participantes com um a sete anos de escolaridade apresentaram correlação moderada positiva do suporte social emocional e do suporte social total com a autoeficácia. Sabe-se que a não alfabetização aliada a poucos anos de educação são fatores que influenciam negativamente a autoeficácia e a adesão ao tratamento. Pessoas com poucos anos de estudo tendem a esquecer de tomar a medicação três a quatro vezes mais do que os que possuem níveis elevados de escolaridade [22], o qual pode ser verificado neste estudo, no qual os participantes com quatro a sete anos de estudo possuem autoeficácia inferior quando comparadas aos outros grupos.

O estigma pode interferir no suporte social, visto que pode desencadear um ciclo: amigos e familiares das pessoas infectadas pelo HIV abandonam o suporte fornecido, tornando-se autores de discriminação [28-29]. Quando os amigos e familiares tornam-se alvos de discriminação por associação, a fonte de suporte social fornecida por estes é inferior, quando comparada à que é fornecida pelos que não sofrem esse tipo de discriminação [23-25]. O estigma interiorizado afeta diretamente o suporte social emocional e está relacionado com o menor grau de instrução, sendo as pessoas menos instruídas mais propensas a endossar crenças populares, de modo que se culpam pela doença e geram avaliações negativas de si $[23,26]$. Assim, o suporte social emocional ajuda a diminuir a legitimidade percebida de avaliações negativas, ajudando as pessoas que vivem com HIV a desenvolverem um sentido mais positivo de si mesmas, levando a uma menor internalização do estigma [23]. A escola é fundamental para o apoio social, por dar início a amizades e possibilitar a ajuda de professores e colegas de aula, o que é evidenciado pela associação entre escolaridade e fonte de apoio fornecida por amigos e outras pessoas.

O menor tempo de diagnóstico e de tratamento apresentaram correlação moderada positiva entre autoeficácia e suporte social emocional. Há evidências de que o suporte social emocional é eficaz principalmente em pessoas que foram infectadas ou descobriram o diagnóstico recentemente [27], tornando-se fundamental no início do diagnóstico, para evitar o estigma interiorizado [23].

Houve correlação entre suporte social emocional e expectativa de autoeficácia em pacientes com menor renda, ou seja, nessas pessoas com renda mais baixa, quanto maior o suporte emocional recebido maior será sua autoeficácia. Essa faixa da população possui médias significativamente superiores de suporte social instrumental e inferiores de expectativa de autoeficácia. Estes dados demonstram que, mesmo havendo uma maior necessidade de suporte instrumental pela condição econômica, o profissional de saúde deve incentivar o suporte emocional, por seu impacto na melhoria da expectativa de autoeficácia.

A correlação verificada entre suporte social emocional e autoeficácia do tratamento em pessoas infectadas pelo HIV com tempo de tratamento ou diagnóstico inferior a cinco anos evidencia que o momento do diagnóstico/início do tratamento é um período que gera inúmeras dúvidas sobre o tratamento e a infecção pelo HIV [28-29], demonstrando a necessidade de ter como fonte de suporte social os profissionais de saúde. O suporte social foi associado com o tempo de diagnóstico, o que pode ser explicado pelo fato de que o maior tempo proporciona ao profissional de saúde 
mais condições de avaliar e implementar intervenções para eficácia do tratamento. Fatores como custos com o transporte e distância do local de tratamento podem levar ao abandono do mesmo [30], devendo o profissional de saúde fornecer todas as informações e suporte necessário para evitar esse abandono.

A correlação entre suporte social emocional e autoeficácia do TARV ocorreu tanto em pacientes com valores baixos de linfócitos T CD4+ quanto naqueles com valores elevados de carga viral. Este fato se justifica pela relação negativa que geralmente ocorre entre linfócitos T CD4 ${ }^{+}$e carga viral [10,28,30-32].

Possuir carga viral indetectável eleva a chance de suporte social, entretanto reduz a expectativa de autoeficácia. Estes achados podem ser explicados pela piora da condição de saúde devida à baixa autoeficácia e à contagem de linfócitos T CD4+, com consequente aumento da carga viral, levando ao agravamento do quadro clínico do paciente e, consequentemente, ao maior suporte social fornecido nesse momento.

A correlação encontrada entre suporte social emocional e autoeficácia, quando colegas de trabalho conheciam a situação de infecção pelo HIV, evidencia que o apoio de colegas de trabalho e familiares incentiva a procura e o acompanhamento adequados aos serviços de saúde, além da redução de sintomas de ansiedade e de depressão [27,31]. Fato este que pode ser verificado pela regressão logística multinomial, pois pessoas que consideram moderadamente difícil ou difícil acessar o serviço de saúde possuem redução da expectativa de autoeficácia. Além disso, com o conhecimento da infecção por colegas de trabalho há aumento da adesão e da autoeficácia do tratamento medicamentoso [32].

Este estudo foi pioneiro, no Brasil, ao avaliar as relações entre suporte social e expectativa de autoeficácia, assim como seus fatores de risco, em pacientes infectados pelo HIV que estavam em TARV há pelo menos três meses. Os resultados obtidos sugerem que o suporte social fornecido e a expectativa de autoeficácia, além de afetar a resposta terapêutica ao tratamento, podem interferir no acompanhamento do serviço de saúde, assim como servir como barreira de proteção para manutenção da adesão ao TARV.

\section{REFERÊNCIAS}

1. World Health Organization. Global report: UNAIDS report on the global AIDS epidemic 2013. Geneva: UNAIDS; 2013.

2. Brasil. Boletim epidemiológico - Aids e DST. Brasília: Ministério da Saúde - Secretaria de Vigilância em Saúde; 2013.

3. Lombardo PG, Costa Aguiar BG. Envejeciendo con AIDS o el AIDS en el envejecimiento: perfil epidemiológico en un hospital de la Universidad de Rio de Janeiro. Enfermería Glob. 2012;11:10-20. http://dx.doi.org/10.4321/S1695-61412012000200002

4. Wasti SP, van Teijlingen E, Simkhada P, Randall J, Baxter S, Kirkpatrick P, Gc VS. Factors influencing adherence to antiretroviral treatment in Asian developing countries: a systematic review. Trop Med Int Health. 2012 Jan;17(1):71-81. http://dx.doi.org/10.1111/j.13653156.2011.02888.x

5. Parienti JJ, Bangsberg DR, Verdon R, Gardner EM. Better adherence with once-daily antiretroviral regimens: a meta-analysis. Clin Infect Dis. 2009 Feb 15;48(4):484-8. http://dx.doi.org/10.1086/596482

6. Merten S, Kenter E, McKenzie O, Musheke M, Ntalasha H, Martin-Hilber A. Patient-reported barriers and drivers of adherence to antiretrovirals in sub-Saharan Africa: a meta-ethnography. Trop Med Int Health. 2010 June;15 Suppl 1:16-33. http://dx.doi.org/10.1111/ j.1365-3156.2010.02510.x

7. Remor E. Systematic review of the psychometric properties of the questionnaire to evaluate the adherence to HIV therapy (CEAT-VIH). Patient. 2013;6(2):61-73. http://dx.doi.org/10.1007/s40271-013-0009-0

8. Chesney MA, Ickovics JR, Chambers DB, Gifford AL, Neidig J, Zwickl B, Wu AW. Self-reported adherence to antiretroviral medications among participants in HIV clinical trials: the AACTG adherence instruments. Patient Care Committee \& Adherence Working Group of the Outcomes Committee of the Adult AIDS Clinical Trials Group (AACTG). AIDS Care. 2000 June;12(3):255-66. http://dx.doi. org/10.1080/09540120050042891

9. Ironson G, Hayward H. Do positive psychosocial factors predict disease progression in HIV-1? A review of the evidence. Psychosom Med. 2008 June;70(5):546-54. http://dx.doi.org/10.1097/PSY.0b013e318177216c

10. Smith R, Rossetto K, Peterson BL. A meta-analysis of disclosure of one's HIV-positive status, stigma and social support. AIDS Care. 2008 Nov;20(10):1266-75. http://dx.doi.org/10.1080/09540120801926977

11. Wolf MS, Davis TC, Osborn CY, Skripkauskas S, Bennett CL, Makoul G. Literacy, self-efficacy, and HIV medication adherence. Patient Educ Couns. 2007 Feb;65(2):253-60. http://dx.doi.org/10.1016/j.pec.2006.08.006

12. Swendeman D, Ingram BL, Rotheram-Borus MJ. Common elements in self-management of HIV and other chronic illnesses: an integrative framework. AIDS Care. 2009 Oct;21(10):1321-34. http://dx.doi.org/10.1080/09540120902803158

13. Leite JC, Drachler ML, Centeno MO, Pinheiro CA, Silveira VL. Desenvolvimento de uma escala de auto-eficácia para adesão ao tratamento anti-retroviral. Psicol Reflex Crit. 2002;15:121-33. http://dx.doi.org/10.1590/S0102-79722002000100014 
14. Seidl EM, Tróccoli BT. Desenvolvimento de escala para avaliação do suporte social em HIV/aids. Psic Teor e Pesq. 2006;22:317-26. http://dx.doi.org/10.1590/S0102-37722006000300008

15. Bekele T, Rourke SB, Tucker R, Greene S, Sobota M, Koornstra J, Monette L, Rueda S, Bacon J, Watson J, Hwang SW, Dunn J, Guenter D; Positive Spaces Healthy Places Team. Direct and indirect effects of perceived social support on health-related quality of life in persons living with HIV/AIDS. AIDS Care. 2013;25(3):337-46. http://dx.doi.org/10.1080/09540121.2012.701716

16. Colbert AM, Kim KH, Sereika SM, Erlen JA. An examination of the relationships among gender, health status, social support, and HIV-related stigma. J Assoc Nurses AIDS Care. 2010 July-Aug;21(4):302-13. http://dx.doi.org/10.1016/j.jana.2009.11.004

17. Cha E, Erlen JA, Kim KH, Sereika SM, Caruthers D. Mediating roles of medication -taking self-efficacy and depressive symptoms on self-reported medication adherence in persons with HIV: a questionnaire survey. Int J Nurs Stud. 2008 Aug;45(8):1175-84. http://dx.doi. org/10.1016/j.ijnurstu.2007.08.003

18. Johnson MO, Neilands TB, Dilworth SE, Morin SF, Remien RH, Chesney MA. The role of self-efficacy in HIV treatment adherence: validation of the HIV Treatment Adherence Self-Efficacy Scale (HIV-ASES). J Behav Med. 2007 Oct;30(5):359-70. http://dx.doi org/10.1007/s10865-007-9118-3

19. Chesney MA, Neilands TB, Chambers DB, Taylor JM, Folkman S. A validity and reliability study of the coping self-efficacy scale. Br J Health Psychol. 2006 Sept;11(Pt 3):421-37. http://dx.doi.org/10.1348/135910705X53155

20. Colombrini MR, Coleta MF, Lopes MH. Fatores de risco para a não adesão ao tratamento com terapia antiretroviral altamente eficaz. Rev Esc Enferm USP. 2008 Sept;42(3):490-5. http://dx.doi.org/10.1590/S0080-62342008000300011

21. López LC. Uma análise das políticas de enfrentamento ao HIV/Aids na perspectiva da interseccionalidade de raça e gênero. Saúde Soc. 2011;20:590-603. http://dx.doi.org/10.1590/S0104-12902011000300006

22. Kalichman SC, Ramachandran B, Catz S. Adherence to combination antiretroviral therapies in HIV patients of low health literacy. J Gen Intern Med. 1999 May;14(5):267-73. http://dx.doi.org/10.1046/j.1525-1497.1999.00334.x

23. Casale M, Wild L, Kuo C. "They give us hope": HIV-positive caregivers' perspectives on the role of social support for health. AIDS Care. 2013;25(10):1203-9. http://dx.doi.org/10.1080/09540121.2013.763893

24. Bogart LM, Cowgill BO, Kennedy D, Ryan G, Murphy DA, Elijah J, Schuster MA. HIV-related stigma among people with HIV and their families: a qualitative analysis. AIDS Behav. 2008 Mar;12(2):244-54. http://dx.doi.org/10.1007/s10461-007-9231-x

25. Yang LH, Kleinman A. 'Face' and the embodiment of stigma in China: the cases of schizophrenia and AIDS. Soc Sci Med. 2008 Aug;67(3):398-408. http://dx.doi.org/10.1016/j.socscimed.2008.03.011

26. Corrigan PW, Watson AC. The Paradox of self-stigma and mental illness. Clin Psychol (New York). 2002;9(1):35-53. http://dx.doi. org/10.1093/clipsy.9.1.35

27. Kelly JD, Hartman C, Graham J, Kallen MA, Giordano TP. Social Support as a Predictor of Early Diagnosis, Linkage, Retention, and Adherence to HIV Care: Results From The Steps Study. J Assoc Nurses AIDS Care. 2014 Sept-Oct;25(5):405-13. http://dx.doi org/10.1016/j.jana.2013.12.002

28. Aragonés C, Sánchez L, Campos JR, Pérez J. Antiretroviral therapy adherence in persons with HIV/AIDS in Cuba. MEDICC Rev. 2011 Apr;13(2):17-23

29. Cooper V, Gellaitry G, Hankins M, Fisher M, Horne R. The influence of symptom experiences and attributions on adherence to highly active anti-retroviral therapy (HAART): a six-month prospective, follow-up study. AIDS Care. 2009 Apr;21(4):520-8. http://dx.doi. org/10.1080/09540120802301824

30. Govindasamy D, Ford N, Kranzer K. Risk factors, barriers and facilitators for linkage to antiretroviral therapy care: a systematic review. AIDS. 2012 Oct 23;26(16):2059-67. http://dx.doi.org/10.1097/QAD.0b013e3283578b9b

31. Liu L, Pang R, Sun W, Wu M, Qu P, Lu C, Wang L. Functional social support, psychological capital, and depressive and anxiety symptoms among people living with HIV/AIDS employed full-time. BMC Psychiatry. 2013 Dec 1;13:324. http://dx.doi.org/10.1186/1471244X-13-324

32. Fuertes JN, Mislowack A, Bennett J, Paul L, Gilbert TC, Fontan G, Boylan LS. The physician-patient working alliance. Patient Educ Couns. 2007 Apr;66(1):29-36. http://dx.doi.org/10.1016/j.pec.2006.09.013 